\title{
Right-sided Infective Endocarditis with a Ruptured Sinus of Valsalva and Multiple Septic Pulmonary Emboli in a Patient with Atopic Dermatitis
}

\author{
Koshin Horimoto ${ }^{1}$, Toshihiko Kubo ${ }^{1}$, Hidenori Matsusaka ${ }^{1}$, \\ Hironori $\mathrm{Baba}^{2}$ and Masayoshi Umesue ${ }^{2}$
}

\begin{abstract}
We herein report the case of 34-year-old woman with acute tricuspid valve infective endocarditis (IE) associated with a ruptured sinus of Valsalva and multiple septic pulmonary emboli. She had no history of medical problems, except for atopic dermatitis (AD). Blood cultures identified methicillin-sensitive Staphylococcus aureus. Despite the administration of two months of antibiotic therapy, the patient experienced recurrent pulmonary emboli and developed heart failure due to a left-to-right shunt, whereas the area of vegetation did not change in size. She subsequently underwent surgery for shunt closure and tricuspid valve replacement. The $\mathrm{AD}$ was thought to be the cause of the patient's bacteremia, which consequently resulted in aggressive rightsided IE.
\end{abstract}

Key words: right-sided infective endocarditis, atopic dermatitis, Valsalva sinus rupture, septic pulmonary embolism

(Intern Med 54: 797-800, 2015)

(DOI: 10.2169/internalmedicine.54.3374)

\section{Introduction}

Right-sided infective endocarditis (IE) is uncommon, accounting for only 5 to $10 \%$ of all cases of IE (1). Atopic dermatitis (AD) is a chronic, relapsing eczematous skin disease. Colonization of Staphylococcus aureus is commonly observed in the skin lesions of AD patients (2), and a relationship between IE and $\mathrm{AD}$ has recently been reported (3-6). However, there are few case reports of rightsided IE in patients with $\mathrm{AD}(4,6)$. We herein report the case of a patient with no predisposing background factors except for AD who suffered from right-sided IE with a ruptured sinus of Valsalva and multiple septic pulmonary emboli. In addition to antibiotic therapy, the patient required surgery for shunt closure and tricuspid valve replacement and subsequently exhibited a good clinical course.

\section{Case Report}

A 34-year-old woman was admitted to our institution for a sustained high fever that had lasted for two weeks. She had no history of medical problems, except for AD since childhood and was not a drug abuser. On admission, her body temperature was 38.6 degrees Celsius and her blood pressure was 106/60 $\mathrm{mmHg}$. She had an elevated white blood cell count (WBC) of $14 \times 10^{9} / \mathrm{L}$ and C-reactive protein (CRP) level of $10.1 \mathrm{mg} / \mathrm{dL}$. No heart murmurs were audible at that time; however, chest computed tomography (CT) showed multiple lung nodules (Fig. 1). Two days after admission, a new continuous 4/6 systolic-diastolic precordial murmur appeared. A transthoracic echocardiogram (TTE) depicted fluttering of a $20 \times 8-\mathrm{mm}$ area of vegetation on the septal leaflet of the tricuspid valve with a turbulent left-toright shunt flow from the Valsalva sinus of a non-coronary cusp to the right atrium through a $5-\mathrm{mm}$ fistula (Fig. 2).

${ }^{1}$ Department of Cardiology, Matsuyama Red Cross Hospital, Japan and ${ }^{2}$ Department of Cardiovascular Surgery, Matsuyama Red Cross Hospital, Japan

Received for publication May 27, 2014; Accepted for publication August 25, 2014

Correspondence to Dr. Toshihiko Kubo, toskub@gmail.com 


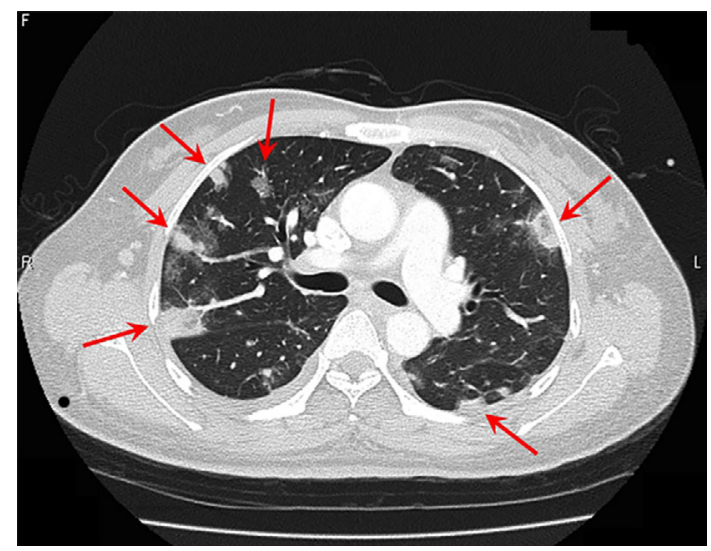

Figure 1. Chest computed tomography scan shows multiple nodules in both lungs (arrows).

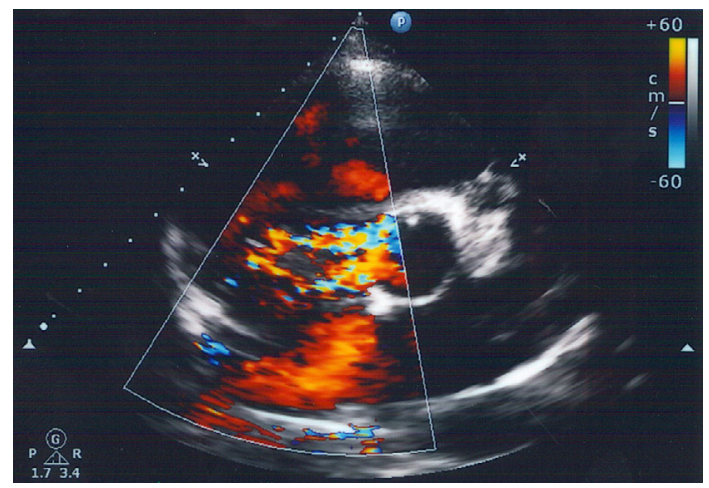

Figure 3. The left-to-right shunt flow slightly increased on a transthoracic echocardiogram.

There was no aortic or tricuspid regurgitation, and the serum brain natriuretic peptide (BNP) level was $568.0 \mathrm{pg} / \mathrm{mL}$. All six sets of blood cultures grew methicillin-sensitive Staphylococcus aureus. The patient was therefore diagnosed as having a ruptured Valsalva sinus and septic pulmonary emboli associated with right-sided IE. Her skin was red and scaly, with many scratches due to uncontrolled AD. There were no scars from intravenous injections.

The patient initially refused surgical intervention. We attempted to keep her skin clean and encouraged her not to scratch with unclean fingers. We also initiated treatment with cefazolin at a dose of $8 \mathrm{~g} /$ day and gentamicin at a dose of $450 \mathrm{mg} /$ day. During the first week of treatment, her symptoms improved and her high fever disappeared. On day 8 , blood cultures became negative; however, the inflammatory reaction remained strongly positive, with a WBC count of $10 \times 10^{9} / \mathrm{L}$ and CRP level of $13.4 \mathrm{mg} / \mathrm{dL}$. According to the antibiotic sensitivity test performed on day 7 , we changed the treatment regimen from gentamicin to levofloxacin at a dose of $500 \mathrm{mg} /$ day. Because the patient began to feel nausea after the infusion of cefazolin on day 23 , cefazolin was changed to imipenem/cilastatin at a dose of $2 \mathrm{~g} /$ day. At that time, the WBC count was $11 \times 10^{9} / \mathrm{L}$ and the CRP level was $7.6 \mathrm{mg} / \mathrm{dL}$. On day 42 , we stopped the imipenem/cilastatin treatment due to nausea and continued the levofloxacin at a

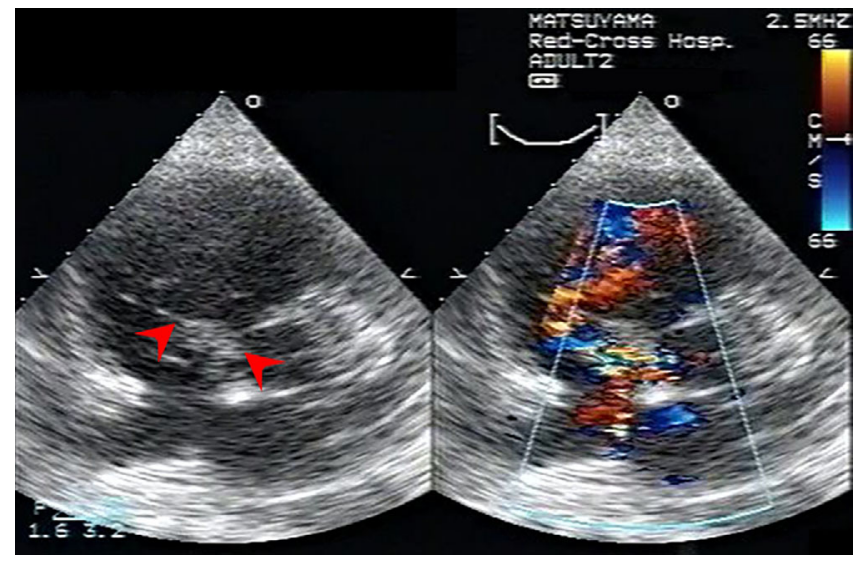

Figure 2. Transthoracic echocardiogram (short-axis view of the aortic valve) depicted a fluttering area of vegetation on the septal leaflet of the tricuspid valve (left, between arrows) and a turbulent flow from the Valsalva sinus of the non-coronary cusp to the right atrium through a fistula (right).

dose of $500 \mathrm{mg} / \mathrm{day}$. Although the area of vegetation remained unchanged on TTE, her body temperature normalized and the inflammatory reaction weakened, with a WBC count of $5 \times 10^{9} / \mathrm{L}$ and CRP level of $1.70 \mathrm{mg} / \mathrm{dL}$ on day 47 . Thereafter, the lung nodules on chest CT disappeared. However, on day 66, although the inflammatory reaction remained stabilized (WBC: $5 \times 10^{9} / \mathrm{L}$ and CRP: $1.10 \mathrm{mg} / \mathrm{dL}$ ), she began to feel general fatigue. In addition, the BNP level decreased to $100.4 \mathrm{pg} / \mathrm{mL}$ on day 24 then subsequently increased to $492.6 \mathrm{pg} / \mathrm{mL}$ and new lung nodules were detected on chest CT. On TTE, the left-to-right shunt volume slightly increased based on a visual inspection (Fig. 3). We therefore initiated medical treatment for heart failure with lisinopril at a dose of $5 \mathrm{mg} /$ day and carvedilol at a dose of $2.5 \mathrm{mg} /$ day, which was gradually increased to $10 \mathrm{mg} /$ day. Finally, the patient underwent surgery for shunt closure and tricuspid valve replacement on day 85 .

During the surgery, a fistula was detected connecting the Valsalva sinus just above the non-coronary aortic cusp and right atrium above the tricuspid valve (Fig. 4A). There were neither ventricular septal defects nor aortic valve lesions; however, a $20 \times 10-\mathrm{mm}$ area of yellow fragile vegetation was noted on the right atrial side of the tricuspid valve septal leaflet (Fig. 4B). Because most of the tricuspid septal leaflet was resected to remove the vegetation, we were unable to perform tricuspid valvuloplasty. We subsequently conducted patch closure of the fistula from the aortic side and tricuspid valve replacement with a biological valve (Perimount 29 $\mathrm{mm}$, Edwards Lifesciences, Irvine, USA). The histological findings of the resected leaflet showed superficial fibrin networks of necrotic debris and granulomatous changes, such as the proliferation of inflammatory cells and microvessels, compatible with the features of IE (Fig. 5). Although no bacterial organisms were detected on histology, Staphylococcus lugdunensis was cultivated from the resected right atrium around the fistula.

After the surgery, the patient received $120 \mathrm{mg} /$ day of gen- 

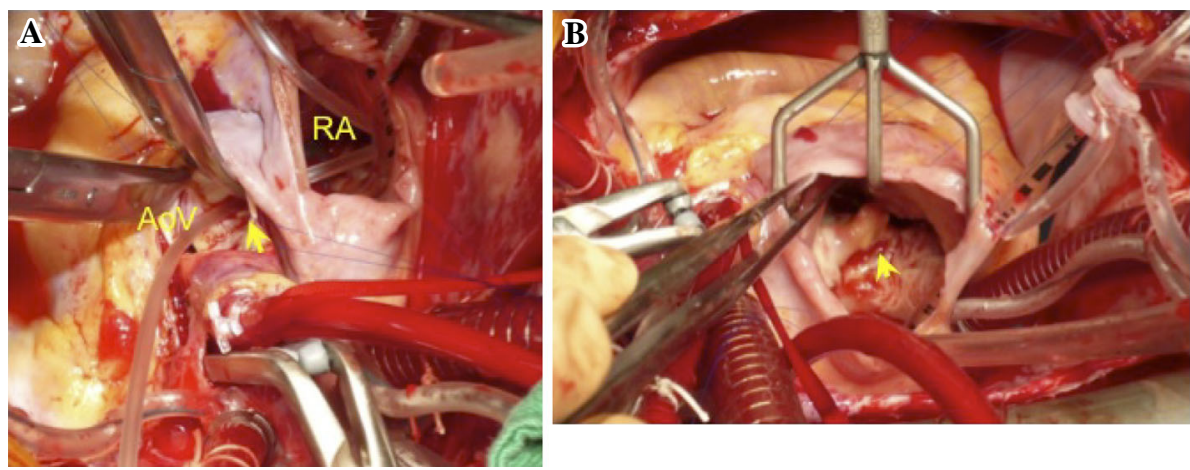

Figure 4. A: A tube was inserted through the fistula (arrow) between the non-coronary sinus of Valsalva and the right atrium (RA). B: A large area of vegetation was located on the septal leaflet of the tricuspid valve (arrow). AoV: aortic valve
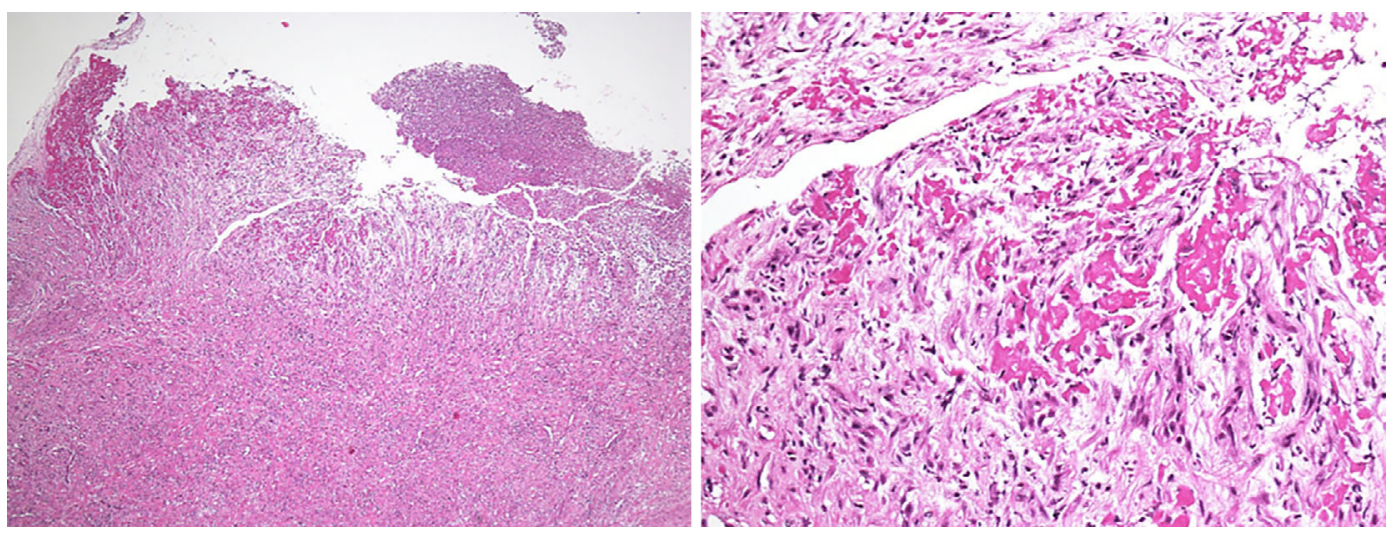

Figure 5. Microscopic findings of the resected leaflet. Left, Hematoxylin and Eosin (H\&E) staining, 40x. Right, H\&E staining, 200x.

tamicin for two weeks and $2 \mathrm{~g} /$ day of imipenem/cilastatin for four weeks. Her clinical course was uneventful, and she was discharged on day 132. Since then, her skin condition has remained stable, with no recurrence of infection or heart failure for 12 months.

\section{Discussion}

Right-sided IE is relatively rare, primarily occurring in patients with a history of intravenous drug use, central venous lines, pacemakers or congenital heart disease (1). Compared with left-sided IE, right-sided IE displays a better response to antibiotic therapy with a reduced need for surgery (1). In the present case, right-sided IE occurred in a patient with no underlying heart disease, including a tricuspid valve, or predisposing background factors, although she had AD. AD is a common skin disease, and the skin of AD patients provides favorable conditions for colonization, proliferation and invasion by Staphylococcus aureus (2). Scratching pruritic lesions may result in repeated bacteremia. Recently, the relationship between IE and AD has received attention (3-6). A Japanese nationwide web-based survey of IE from 2007 to 2009 identified AD as a risk factor for IE; AD accounted for $1.2 \%$ of predisposing back- ground factors for IE (7). Fukunaga et al. evaluated the early and late outcomes of eight patients with IE and $\mathrm{AD}$ (5). In that report, only one patient showed right-sided IE with tricuspid regurgitation. To the best of our knowledge, this is the first report in which right-sided IE was associated with Valsalva sinus rupture and multiple septic pulmonary emboli in a patient with AD.

In the present case, two different organisms were detected: methicillin-sensitive Staphylococcus aureus from blood cultures obtained on admission and Staphylococcus lugdunensis from the culture of the resected right atrium during surgery. Both of these microbes are aggressive staphylococci and commonly induce valvular destruction and abscess formation, resulting in acute and sometimes fatal clinical courses (1). Staphylococcus lugdunensis is a coagulase-negative staphylococcus and component of the normal human skin flora (8). Compared with infections caused by other coagulase-negative staphylococci and even Staphylococcus aureus, Staphylococcus lugdunensis endocarditis follows a more aggressive and complicated clinical course (8). In the present case, we believe that the patient's scratching of her pruritic AD skin lesions induced bacteremia with methicillin-sensitive Staphylococcus aureus, which colonized the tricuspid valve, leading to the accumulation of 
vegetation and formation of multiple septic pulmonary emboli that subsequently invaded backward to the right atrial septum and finally resulted in the construction of the fistula to the Valsalva sinus. Most of Staphylococcus aureus was eradicated with aggressive antibiotic therapy, and Staphylococcus lugdunensis was detected following microbial substitution. Fortunately, the administration of additional antibiotic therapy after surgery controlled the infection.

The patient exhibited a ruptured sinus of Valsalva and multiple pulmonary emboli two days after hospital admission, and echocardiography demonstrated vegetation measuring larger than $10 \mathrm{~mm}$. These signs indicate the need for early surgical intervention (1). However, she was reluctant to undergo urgent surgery, and surgical intervention was considered to be dangerous based on her status of uncontrolled sepsis. Because a large area of vegetation occupied the tricuspid septal leaflet, tricuspid valve replacement was required, which could have resulted in catastrophic prosthetic valve endocarditis. According to the European Society of Cardiology guidelines, surgical treatment should generally be avoided in patients with right-sided native IE, although it should be considered in the following situations: a) rightsided IE secondary to severe tricuspid regurgitation with a poor response to diuretic therapy; b) IE caused by organisms that are difficult to eradicate or bacteremia for at least seven days despite adequate antibiotic therapy; c) tricuspid valve vegetation larger than $20 \mathrm{~mm}$ that persists after recurrent pulmonary emboli formation with or without concomitant right heart failure (1). Although the present patient showed large tricuspid valve vegetation and a small Valsalva sinus fistula on admission, she exhibited no signs of heart failure and initially demonstrated a good response to antibiotic therapy. However, despite two months of medical therapy, the area of vegetation did not change in size and she developed recurrent pulmonary emboli with mild heart failure associated with a gradually increasing left-to-right shunt. Therefore, we finally decided to perform elective surgical treatment before her general condition worsened.

Because the sinus of Valsalva was involved, the present case may include "left-sided" IE not simply "right-sided" IE. Fistula formation with "left-sided" native valve IE can cause "refractory heart failure," considered to be due to "uncontrolled infection," and may be an indication for early surgical correction (1). A ruptured sinus of Valsalva complicated by IE may result in an extremely serious condition depending on the shunt volume, and emergent or urgent surgery is required if heart failure worsens rapidly. According to the findings of a large cohort study of 76 patients with aorto-cavitary fistulous tract formation complicated by IE, 22 patients underwent elective surgery, while surgery was delayed for $>30$ days in six patients. Meanwhile, 10 patients did not undergo surgery, seven of whom survived. In that cohort, emergent or urgent surgery was the independent risk factor for in-hospital mortality (9). In the present case, although we did not measure the Qp/Qs on echocardiograms, the left-to-right shunt volume was not large based on a visual inspection and no right-sided volume overload was evident throughout the patient's clinical course. Fortunately, she did not require urgent surgery and was able to observe a three-week waiting period. Because her heart failure worsened slowly with medical treatment, she had time to get herself into a good mental condition during the waiting period.

Generally, right-sided IE displays a good response to medical therapy. However, surgical management is sometimes necessary depending on the complications. Hence, physicians should strictly evaluate the patient's general conditions and determine the optimal timing for surgery when required.

The authors state that they have no Conflict of Interest (COI).

\section{References}

1. Habib G, Hoen B, Tornos P, et al. Guidelines on the prevention, diagnosis, and treatment of infective endocarditis (new version 2009): the Task Force on the Prevention, Diagnosis, and Treatment of Infective Endocarditis of the European Society of Cardiology (ESC). Endorsed by the European Society of Clinical Microbiology and Infectious Diseases (ESCMID) and the International Society of Chemotherapy (ISC) for Infection and Cancer. Eur Heart J 30: 2369-2413, 2009.

2. Hauser C, Wuethrich B, Matter L, Wilhelm JA, Sonnabend W, Schopfer K. Staphylococcus aureus skin colonization in atopic dermatitis patients. Dermatologica 170: 35-39, 1985.

3. Onoda K, Mizutani H, Komada T, et al. Atopic dermatitis as a risk factor for acute native valve endocarditis. J Heart Valve Dis 9: 469-471, 2000.

4. Pike MG, Warner JO. Atopic dermatitis complicated by acute bacterial endocarditis. Acta Paediatr Scand 78: 463-464, 1989.

5. Fukunaga N, Okada Y, Konishi Y, Murashita T, Koyama T. Pay attention to valvular disease in the presence of atopic dermatitis. Circ J 77: 1862-1866, 2013.

6. Fukumitsu K, Suzuki Y. A septic pulmonary embolism associated with right-sided infective endocarditis and a ventricular septal defect in a patient with atopic dermatitis. Kansenshogaku Zasshi (The Journal of the Japanese Association for Infectious Diseases) 86: 282-286, 2012 (in Japanese, Abstract in English).

7. Nakatani S, Mitsutake K, Ohara T, et al. Recent picture of infective endocarditis in Japan: lessons from Cardiac Disease Registration (CADRE-IE). Circ J 77: 1558-1564, 2013.

8. Zinkernagel AS, Zinkernagel MS, Elzi MV, et al. Significance of Staphylococcus lugdunensis bacteremia: report of 28 cases and review of the literature. Infection 36: 314-321, 2008.

9. Anguera I, Miro JM, Vilacosta I, et al. Aorto-cavitary fistulous tract formation in infective endocarditis: clinical and echocardiographic features of 76 cases and risk factors for mortality. Eur Heart J 26: 288-297, 2005.

(C) 2015 The Japanese Society of Internal Medicine http://www.naika.or.jp/imonline/index.html 